Revue d'histoire de l'Amérique française

\title{
Le suicide dans les enquêtes du coroner au Québec entre 1763 et 1986 : un projet de recherche inédit
}

\author{
Patrice Corriveau, Isabelle Perreault, Jean-François Cauchie et Annie Lyonnais
}

Volume 69, numéro 4, printemps 2016

URI : https://id.erudit.org/iderudit/1036515ar

DOI : https://doi.org/10.7202/1036515ar

Aller au sommaire du numéro

\section{Éditeur(s)}

Institut d'histoire de l'Amérique française

\section{ISSN}

0035-2357 (imprimé)

1492-1383 (numérique)

Découvrir la revue

\section{Citer cette note}

Corriveau, P., Perreault, I., Cauchie, J.-F. \& Lyonnais, A. (2016). Le suicide dans les enquêtes du coroner au Québec entre 1763 et 1986 : un projet de recherche inédit. Revue d'histoire de l'Amérique française, 69(4), 71-86.

https://doi.org/10.7202/1036515ar
Résumé de l'article

Cette note de recherche porte sur la création d'une base de données qui rassemblera au final toutes les enquêtes du coroner qui ont conclu à un décès par suicide sur le territoire du Québec, et ce, de la Conquête britannique (1763) à 1986, soit plus ou moins 20000 enquêtes. Riche de matériaux aussi précieux que des rapports médicaux, des témoignages de proches ou de professionnels d'horizons divers, des verdicts de jury et coroner ou encore des lettres d'adieu, cette base de données ne permettra pas seulement des analyses de type épidémiologique. Elle ouvrira aussi la voie à l'examen de l'évolution des discours sociaux qui accompagnent le geste suicidaire. Nous aborderons également le fait que la création et l'utilisation d'une telle base de données ne viennent pas sans leur lot de dilemmes épistémologiques et méthodologiques.
Tous droits réservés @ Institut d’histoire de l’Amérique française, 2016

Ce document est protégé par la loi sur le droit d'auteur. L'utilisation des services d'Érudit (y compris la reproduction) est assujettie à sa politique d'utilisation que vous pouvez consulter en ligne.

https://apropos.erudit.org/fr/usagers/politique-dutilisation/ 




\begin{abstract}
RÉSumÉ - Cette note de recherche porte sur la création d'une base de données qui rassemblera au final toutes les enquêtes du coroner qui ont conclu à un décès par suicide sur le territoire du Québec, et ce, de la Conquête britannique (1763) à 1986, soit plus ou moins 20000 enquêtes. Riche de matériaux aussi précieux que des rapports médicaux, des témoignages de proches ou de professionnels d'horizons divers, des verdicts de jury et coroner ou encore des lettres d'adieu, cette base de données ne permettra pas seulement des analyses de type épidémiologique. Elle ouvrira aussi la voie à l'examen de l'évolution des discours sociaux qui accompagnent le geste suicidaire. Nous aborderons également le fait que la création et l'utilisation d'une telle base de données ne viennent pas sans leur lot de dilemmes épistémologiques et méthodologiques.
\end{abstract}

ABSTRACT - This research note focuses on the creation of a database that will eventually include all 20000 coroners' inquests conducted in Quebec from the British Conquest (1763) to 1986 that have returned a verdict of death by suicide. With its invaluable material ranging from medical records, witness statements from relatives and diverse professionals, coroner

1. Cette recherche bénéficie d'une subvention du Conseil de recherche en sciences humaines du Canada (CRSHC) et du Réseau québécois sur le suicide, les troubles de l’humeur et les troubles associés (RQSHA). 
and jury verdicts to farewell letters, the relevance of this database will not be limited to epidemiological analyses. It will also facilitate a better understanding of the evolution of social discourses on suicidal gestures and will shed some light, through their own words, on the lives of those who chose to put an end to their existence. The note also addresses the fact that the creation and use of such a database does not come without its share of thorny epistemological and methodological dilemmas.

Celui qui a été ne peut plus désormais ne pas avoir été : désormais ce fait mystérieux et profondément obscur d'avoir vécu est son viatique pour l'éternité ${ }^{2}$.

Jankélévitch, Penser la mort? (1983)

M ême si le suicide fait depuis longtemps l'objet de préoccupations, ce n'est que depuis une quarantaine d'années qu’un peu partout au Canada et notamment au Québec, les autorités publiques intensifient leurs interventions en ce qui concerne les décès par suicide ${ }^{3}$. La mise sur pied en 1979 d'un groupe d'étude national sur le suicide par le ministère de Santé et Bien-être Canada témoigne d'un intérêt plus marqué pour comprendre les décès attribués à un suicide ${ }^{4}$. Parallèlement à cette initiative, on assiste dans le milieu universitaire à la naissance d'un nouveau champ de recherche et d'intervention spécifiquement axé sur ce phénomène: la suicidologie $\mathrm{e}^{5}$. On tente plus que jamais de comprendre et d'expliquer les causes du passage à l'acte suicidaire, qu'elles soient d'ordre sociologique, psychologique, médical, biologique ou autres.

2. Vladimir Jankélévitch, "L’irréversible et la Nostalgie», dans Penser la mort? (Paris, Liana Levi, [1983] 1994), 13.

3. À noter que nous employons ici le terme suicide comme un "générique» englobant les verdicts en lien avec le fait de s'enlever la vie comme ceux de felo de se, non compos mentis, destruction personnelle, destruction volontaire, suicide, s'est pendu lui-même, hanged himself, mort violente sans responsabilité criminelle (suicide), etc.

4. Deux volumineux rapports intitulés Le suicide au Canada (1987 et 1994) en découleront.

5. Marie-France Aujard, Genèse et nature de la suicidologie, thèse de doctorat (sociologie), Université Laval, 2008; Edwin S. Shneidman, "A Psychological Approach to Suicide», dans G. Vandebos et al., dir. Cataclysms, Crises and Catastrophes: Psychology in Action (American Psychological Association, 1987), 151183. En 1987 on voit naître notamment au Québec l'Association québécoise de suicidologie. Par la suite, plusieurs groupes de recherche importants dont le CRISE (Centre de recherche et d'intervention sur le suicide et l'euthanasie) à l'UQAM ou la chaire de suicidologie Arthur Sommer Rotenberg à Toronto ont vu le jour au Canada. 
Aujourd'hui encore, la recherche sur le suicide s'inscrit essentiellement dans le "paradigme scientifique traditionnel, caractérisé par le souci de prédire, de contrôler et de comprendre $[. .$.$] à l'intérieur de modèles de$ recherche qui sont d'ordinaire de nature quantitative ${ }^{6} »$. La dimension épidémiologique centrée sur les facteurs de risque et de protection prédomine en effet dans la littérature ${ }^{7}$. Bien que ces recherches informent sur l'évolution des taux de suicides ainsi que sur leurs corrélations avec certains facteurs sociodémographiques ou encore tentent de mieux saisir les facteurs suicidogènes, elles ne rendent pas compte de l'évolution, sur un temps long, des diverses sensibilités par rapport au geste suicidaire et des conjonctions/ tensions institutionnelles et disciplinaires observées dans sa définition et sa prise en charge. Plusieurs chercheurs reconnaissent par ailleurs l'importance des démarches plus compréhensives qui permettent de reconstruire le parcours de vie des personnes avant et au moment de s'enlever la vie, et ce, à partir des enquêtes du coroner, des entretiens semi-dirigés avec les proches ou encore des documents personnels comme les lettres d'adieu, etc. L'intérêt d'étudier des documents de première main comme les lettres d'adieu, afin de mieux saisir les motivations et les causes présumées du passage à l'acte, est largement démontré par la littérature ${ }^{8}$.

Notre équipe de recherche a retracé à cet égard des corpus documentaires qui permettent à la fois d'offrir une analyse soutenue et suivie de l'évolution des discours et des comportements/réactions par rapport au suicide sur près de 250 ans, mais aussi d'éclairer des parcours ou des fins de vie anonymes racontés par la plume de ceux-là mêmes qui ont choisi d'en témoigner. L'étude s'inscrit sur le territoire géographique du Québec depuis la Conquête. C’est à partir des années 1760 que les enquêtes du coroner, officiers du système judiciaire britannique, prennent forme dans la colonie. À partir de traces composées de témoignages des proches et de décisions des autorités judiciaires, il est possible de mettre à l'épreuve

6. Recherche sur le suicide au Canada: Aperçu descriptif, Centre de recherche sur le suicide et l'euthanasie (CRISE) (2003), 36.

7. Voir notamment l'article et les références de Alain Lesage et al., «Perspectives de la santé publique pour la prévention du suicide», Santé mentale au Québec, 37, 2 (2012): 239-255.

8. De manière non exhaustive, voir les travaux Francine Gratton avec la coll. de Jacques Lazure, Les suicides d'être de jeunes Québécois (Québec, Presses de l'Université du Québec, 1996); Anton A. Leenaars, "Suicide notes in the courtroom», Journal of Clinical Forensic Medicine, 6 (1999): 39-48; John P. Pestian, Pawel Matykiewicz et Michelle Linn-Gust, "What's in a Note: Construction Of A Suicide Note Corpus », Biomedical Informatics Insights, 5 (2012): 1-6; Sandra Sanger et Patricia McCarthy Veach, «The Interpersonal Nature of Suicide: A Qualitative Investigation of Suicide Notes ", Archives of Suicide Research, 12 (2008): 352-365 ; Éric Volant, Adieu la vie... Étude des derniers messages laissés par les suicidés (Québec, Bellarmin, 1990); Bijou Yang et David Lester, "The Presentation of the Self : An Hypothesis about Suicide Notes », Suicidology Online, 2 (2011): 75-79. 
du temps un phénomène social, le suicide, mais aussi ce geste très personnel qu'est le fait de se donner la mort.

La difficulté d'en rendre compte sur la longue durée a été constatée par Georges Minois dans son Histoire du suicide $e^{9}$. Convaincu de la nécessité de disposer des archives judiciaires pour mieux comprendre le geste suicidaire, Minois souligne que ces archives sont souvent très fragmentaires et que pour les reconstituer, il faut généralement «avoir recours à des sources variées, hétéroclites et peu abondantes ${ }^{10}$ ». John Weaver, lui, insiste sur la pertinence d'étudier des sources judiciaires comme les enquêtes du coroner, archives qu'il a dépouillées et analysées dans le cadre de ces deux plus récentes monographies ${ }^{11}$. Mentionnons aussi que des chercheurs comme Howard Kushner aux États-Unis, Joseph Merrick et Dominique Godineau en France, Janet Miron en Ontario, John Weaver en Nouvelle-Zélande et au Queensland (Australie) ou encore Michael MacDonald et Terence Murphy ainsi que C. Jennings et Brian Baraclought en Angleterre ont également mobilisé les archives judiciaires pour étudier le geste suicidaire sur la longue durée ${ }^{12}$.

Au Québec, la plupart des recherches menées à partir des enquêtes du coroner ont donné lieu à des mémoires de deuxième cycle universitaire ${ }^{13}$. Parallèlement à ces études, notre équipe de recherche a entrepris d'ac-

9. Georges Minois, Histoire du suicide. La société occidentale face à la mort volontaire (Paris, Fayard, 1995).

10. Ibid., 9. Mentionnons que Minois, historien français, ne peut compter sur les enquêtes du coroner, enquêtes de tradition britannique.

11. John C. Weaver, A Sadly Troubled History. The Meanings of Suicide in the Modern Age (Montréal, McGill-Queen's University Press, 2009); John C. Weaver, Sorrows of a Century. Interpreting Suicide in New Zealand, 1900-2000 (Montréal et Kingston, McGill-Queen’s University Press, 2014).

12. Howard I. Kushner, American Suicide. A Psychocultural Exploration (New Brunswick et Londres, Rutgers University Press, 1991); Joseph Merrick, "Death and Life in the Archives: Patterns of and Attitudes to Suicide in Eighteenth-Century Paris ", dans J. C. Weaver et D. Wright, dir., Histories of Suicides. International Perspectives on Self-Destruction in the Modern World (Toronto, University of Toronto Press, 2009): 73-90; Dominique Godineau, S'abréger les jours. Le suicide en France au XVIII siècle (Paris, Armand Colin, 2012); Janet Miron, «Suicide, Coroner's Inquests, and the Parameters of Compassion in Ontario, 1830-1900 ", Histoire sociale/Social History, 47, 95 (novembre 2014) : 577-599; J. C. Weaver, Sorrows of a Century... op. cit. ; J. Weaver, A Sadly Troubled History..., op. cit. ; Michael MacDonald et Terence R. Murphy, Sleepless Souls. Suicide in Early Modern England (Oxford, Clarendon Press, 1990); C. Jennings et Brian Barraclough, «Legal and Administrative Influences on the English Suicide Rate since 1900 », Psychological Medecine, 10 (1980): 407-418.

13. François Bordeleau, «Le suicide dans le district judiciaire de Montréal de 1815 à 1843 et de 1892 à 1903 », mémoire de maîtrise (histoire), UQAM, 2001; Élise Chapdelaine, "Des menottes par-dessus les pansements. Le crime de tentative de suicide dans les tribunaux du Québec de 1892 à 1972 : l'évolution des discours juridique et médical", mémoire de maîtrise (criminologie), Université d'Ottawa, 2011; Michel Sharpe, «La mort violente à Sherbrooke de 1901 à 1930. L'accident mortel, le suicide et l'homicide", mémoire de maîtrise (histoire), Université de Sherbrooke, 1993; Nathalie Ricard, Risque, droit et société : les enquêtes du coroner du district judiciaire de Trois-Rivières, 1850-1950, mémoire de maîtrise (études québécoises), Université du Québec à Trois-Rivières, 2013; André Lachance, «Le suicide», dans Étude sur la mort violente dans les Cantons de l'Est, 1900-1950 (Sherbrooke, Éditions GGC, 2002): 63-97. 
complir depuis 2007 le dépouillement systématique des enquêtes du coroner sur le territoire du Québec ayant conclu à un décès par suicide entre 1763 et $1986^{14}$. Par cette note de recherche, nous voulons présenter notre base de données et rendre compte des enjeux épistémologiques et méthodologiques liés à nos données empiriques.

\section{MISE EN CONTEXTE ET DILEMME ÉPISTÉMOLOGIQUE}

Il existe différentes façons de concevoir le geste suicidaire, qui ne sont pas nécessairement mutuellement exclusives. Elles obligent néanmoins les chercheurs à se positionner épistémologiquement face à la lecture de leurs données. Le suicide sera analysé soit comme "un fait brut», soit comme un "événement social rapporté». Pour les chercheurs qui conçoivent le suicide comme un fait brut, objectivé et objectivable, ce sont généralement les causes du passage à l'acte suicidaire qui les interpellent, que ces causes se situent sur un registre plus global ou davantage individuel ${ }^{15}$. Pour les tenants du suicide comme fait social rapporté par contre, le suicide est d'abord et avant tout une construction discursive: pour eux, les sens donnés au geste suicidaire prennent donc forme dans la parole, les mots et les gestes des acteurs sociaux, et la compréhension du suicide est nécessairement imprégnée du sens que les institutions de contrôle lui attribuent ${ }^{16}$. Entre ces deux paradigmes du suicide, les frontières peuvent être plus perméables qu'il n'y paraît: d'une part, s'intéresser aux causes du suicide n'interdit pas de prêter attention $\mathrm{au}(\mathrm{x})$ sens que les divers acteurs qu'il mobilise lui donnent; et d'autre part, s'intéresser aux diverses réactions sociales que suscite le suicide n'interdit pas sa définition objective préalable.

Étudier les suicides est quoi qu'il en soit une tâche complexe, notamment lorsque cette étude s'étend sur un temps long. Le suicide a en effet revêtu plusieurs visages tout au long de son histoire. De crime de lèse-

14. Voir http://sciencessociales.uottawa.ca/suicide/, notamment pour comprendre l'évolution de notre programme de recherche.

15. Voir par exemple la définition du suicide selon Durkheim: «tout cas de mort qui résulte directement ou indirectement d'un acte positif ou négatif, accompli par la victime elle-même et qu'elle savait devoir produire ce résultat». Émile Durkheim, Le suicide (Paris, Presses universitaires de France, [1897] 2007): 5. Précisons ici que des chercheurs peuvent très bien accepter l'idée que les causes du suicide soient globalement socio-pathologiques mais estimer que pour comprendre ces passages à l'acte sur un registre plus individuel, il faut en appeler à des lectures psycho voire bio-pathologiques (la détérioration de la santé de l'individu trouverait en quelque sorte son origine dans une dégradation sociale).

16. Voir notamment Jack D. Douglas, The Social Meanings of Suicide (Princeton, Princeton University Press, 1967); Ian Marsh, Suicide. Foucault, History and Truth (Cambridge (UK), Cambridge University Press, 2010). 
majesté divine jusqu'au premier tiers du XIX ${ }^{e}$ siècle à enjeu de santé publique aujourd'hui en passant par celui de problème individuel lié à la santé mentale, la façon de concevoir ce geste a grandement changé au fil des siècles ${ }^{17}$. Non seulement le fait de se donner la mort peut être vu à travers des lunettes pénales, religieuses ou médicales, mais la tâche se complique aussi par le fait que les chercheurs ne peuvent s'en remettre à une position nominaliste quand vient le temps de répertorier les cas de suicides à étudier. Selon les époques et selon les coroners chargés de l'enquête, le terme "suicide" n'est en effet qu'une des diverses manières de nommer le fait de s'enlever la vie. Comme nous l'avons déjà mentionné en début d'article, des verdicts aussi divers que felo de se, non compos mentis, mort par pendaison, destruction personnelle ou encore suicide dans un moment de folie se retrouvent dans les plumitifs et dans les verdicts des enquêtes du coroner. Il faut aussi accepter qu’une part de la « réalité empirique» restera inaccessible, que ce soit pour des raisons d'accès aux sources ou encore pour des raisons liées à l'interprétation du geste.

Nous avons ainsi pris soin de colliger les dossiers de décès qui ont été rapportés en tant que suicides au sens large du point de vue du coroner (ou du jury). Une lecture plus attentive est parfois nécessaire pour saisir s'il s'agit bien de suicides du point de vue des acteurs judiciaires et des jurés. Par exemple, un dossier avec un verdict tel que «il s'est tranché la gorge» entrera dans notre base de données même si le terme suicide n'apparaît pas au dossier. Alors que certains coroners s'en remettent essentiellement à l'étiquette "suicide» pour décrire le type de décès, d'autres préfèrent l'appellation répandue "suicide dans un moment de folie» ou encore "mort dans un moment de dérangement/ découragement», alors que d'autres encore optent pour le moyen qui a permis le décès sans autre mention (noyade, asphyxie, pendaison, etc. $)^{18}$.

La posture épistémologique qui nous guide pour élaborer notre corpus empirique et notre base de données est celle qui consiste à considérer le suicide comme un fait social rapporté et non pas comme un fait brut (posture réaliste). Suivant cette perspective théorique, le phénomène du

17. Selon l'historien Georges Minois, le suicide était considéré comme un crime de lèse-majesté divine parce qu'il allait à l'encontre de la vie. Selon la Bible, mettre fin à ses jours délibérément est un blasphème. Dieu seul peut décider de la vie et de la mort des croyants. Le droit canon sera explicite sur l'interdiction de se donner la mort à partir du $\mathrm{XI}^{e}$ et XII' siècles. Voir notamment l'ouvrage de G. Minois, Histoire du suicide..., op. cit., 43-46.

18. Voir par exemple notre article sur les suicides en milieu carcéral : Patrice Corriveau, Jean-François Cauchie et Isabelle Perreault, «Enjeux autour de la responsabilité du geste suicidaire en institution carcérale. Analyse des enquêtes du coroner de Montréal entre 1892 et 1950 ", Champ pénal/Penal field [En ligne], (11, 2014), mis en ligne le 28 novembre 2014. 
suicide ne peut pas être appréhendé indépendamment des processus socioculturels à travers lesquels il est inévitablement "construit» socialement et institutionnellement ${ }^{19}$. Par exemple, à l'instar de Neeleman et Wessely, Pescosolido et Mendelsohn ou encore de Clarke-Finnegan et Fahy, nous constatons dans nos données que le type de formation (juridique ou médicale) reçu par un coroner joue sur la justification du verdict prononcé (décès naturel, meurtre, accident, suicide, etc. ${ }^{20}$. C'est dire que le comportement suicidaire ne peut prendre socialement forme qu'à condition d'être nommé et interprété par les acteurs sociaux concernés, qu'il s'agisse des autorités amenées à devoir qualifier le geste comme suicide, des proches appelés à témoigner sur l'événement ou encore de ceux qui ont eux-mêmes fait le geste ${ }^{21}$. Le défi est d'autant plus ardu que le rôle du coroner change entre 1763 et 1986 . De porte d'entrée du système pénal qu'il était pendant deux siècles, il deviendra au cours des dernières décennies un promoteur de la sécurité publique ${ }^{22}$.

Enfin, mentionnons que le format des enquêtes se modifie au cours de la période, le changement majeur d'inscription des suicides tels que rapportés par le coroner se situant dans les années qui précèdent la décriminalisation de la tentative de suicide au Canada en 1972. Alors qu'au XIX ${ }^{\mathrm{e}}$ siècle, les enquêtes de la Cour du coroner sont entièrement manuscrites et contiennent la liste des jurés assermentés, les transcriptions des témoignages, le verdict et la preuve, à partir de 1914, un nouveau format apparaît, et ce, jusqu'en 1967. L'année 1914 marque en effet une rupture dans les manières de faire ${ }^{23}$. Alors qu'auparavant une enquête avec jurés était tenue systématiquement dans les cas de décès catégorisés violents (accidents, suicides, homicides), à partir de 1914, les rapports du coroner sans

19. Deborah Lupton, Risk (New York, Routledge, 1999), 35.

20. Jan Neeleman et Simon C. Wessely, "Changes in Classification of Suicide in England and Wales: Time Trends and Associations with Coroner's Professional Background», Psychological Medicine, 27 (1997): 467-472; Bernice A. Pescosolido et Robert Mendelsohn, «Social Causation or Social Construction of Suicide? An Investigation into the Social Organization of Official Rates ", American Sociological Review, 51, 1 (1986): 80-100; Mary Clarke-Finnegan et T. J. Fahy, «Suicide Rates in Ireland», Psychological Medecine, 13, (1983), 385-391.

21. Jack Douglas, par exemple, a étudié les différentes significations données au suicide et à la tentative de suicide pour comprendre l'évolution de la réaction sociale à leur égard. Voir J. D. Douglas, The Social Meanings of Suicide..., op.cit.

22. Sur le métier de coroner au Québec, voir notamment Rénald Lessard et Stéphanie Tésio, «Les enquêtes des coroners du district de Québec, 1765-1930: une source en histoire médicale et sociale canadienne», Bulletin canadien d'histoire de la médecine, 25, 2 (2008): 433-460; N. Ricard, Risque, droit et société... op. cit.

23. Bill 5 relatif aux coroners, 4, George V, Chap. 38, 1914, Art. 3487 à 3487qq. 
jurés deviennent légion ${ }^{24}$. Cette manière de faire sera maintenue jusqu'à l'entrée en vigueur de la nouvelle loi des coroners de $1967^{25}$. À partir de cette date, les enquêtes sont non seulement dactylographiées mais classées dans des chemises distinctes (et non plus pliées en quatre dans des boîtes). Une série de nouveaux formulaires peuvent alors y être trouvés. Parmi les principaux, il y a le Procès-verbal du coroner en cas de recherches, Disposition d'un cadavre, Inventaire des objets trouvés sur un cadavre, Déposition d'un témoin, Examen interne ou externe du cadavre, Rapport général et/ou d'incident - service de police, et enfin la ou les lettres de suicide écrite par la personne elle-même s'il y a lieu ${ }^{26}$.

\section{LA CUEILLETTE DES DONNÉES ET LA MISE EN PLACE D'UNE BASE DE DONNÉES}

Nos données numérisées proviennent des Enquêtes du coroner, lesquelles sont conservées dans tous les centres régionaux de Bibliothèque et Archives nationales du Québec (BAnQ): Montréal, Québec, Trois-Rivières, Sherbrooke, Gatineau, Rouyn-Noranda, Saguenay, Sept-Îles, Rimouski et Gaspé. Plus spécifiquement, nous dépouillons le fonds Cour des sessions de la paix (TP12), le fonds Cour de Magistrat (la cote d'archive varie selon les districts), le fonds Cour du banc du roi (TP9) et le fonds Cour des sessions générales de la paix (TL31). À ce jour, notre équipe de recherche a repéré et photographié une bonne partie des enquêtes dont le verdict renvoie explicitement au geste suicidaire. Nous avons près de 17000 dossiers repérés à la BAnQ Montréal (12 258), à la BAnQ Québec (2784), à la BAnQ Sherbrooke (852) et à la BAnQ Gatineau (1003). Le travail de dépouillement se poursuit aux BAnQ de Trois-Rivières, Rouyn-Noranda, Saguenay, Sept-Îles, Rimouski et Gaspé. À terme, ce seront près de 20000

24. Dans le nouveau formulaire, on peut lire ceci: «Rapport au sujet de la mort de ... arrive le ... à ... circonstances de la mort telles que données par ... comme mort...». «Les recherches établissent que cette mort est due à une cause ... qu'elle n'est imputable à crime à qui que ce soit, ni à la négligence de personne; qu'aucun crime ne l'a accompagnée ou précédée et qu'il n'y a pas lieu de tenir une enquête avec jury".

25. Loi des coroners, 16, Eliz. II. 1967, Chap. 19, Art. 34 et 35.

26. Dans les cas de décès suspects ou encore dans les cas d'homicide(s)/suicide, une enquête a lieu. On retrouve alors, en plus des formulaires cités, un avis du coroner concernant la tenue d'une enquête, les informations du coroner données aux personnes présentes lors d'une enquête, le procès-verbal et le verdict concernant une enquête du coroner, l'enquête à proprement parler et les documents de l'Institut de médecine légale et de la police scientifique. 
enquêtes du coroner ayant conclu à un suicide et menées au Québec entre 1763 et $1986^{27}$ qui composeront notre corpus empirique ${ }^{28}$.

Alors que certains dossiers contiennent un ou deux documents, d'autres peuvent être constitués d'une vingtaine d'éléments d'enquêtes. Parmi les informations que l'on peut retrouver dans une enquête du coroner, il y a le rapport médico-légal, les témoignages des proches du décédé et des témoins de l'événement, le rapport d'enquête de la police, la lettre de suicide s'il y a lieu, la liste des jurés de même que le verdict rendu ${ }^{29}$. Le coroner s'attardera à présenter au jury la preuve faisant état du type de décès qu'il interprète au vu des derniers jours et heures de vie du défunt. C'est à partir de ces témoignages que nous seront à même d'analyser la réaction des principaux acteurs sociaux intervenant dans l'enquête.

Devant la qualité et la quantité inestimables de ces données, qui permettront des analyses en tout genre (sociologique, historique, démographique, littéraire, criminologique, épidémiologique, etc.), nous avons décidé de mettre notre base de données en ligne. Sur le plan organisationnel, cette démarche devenait un impératif, car nous devions nous doter d'un outil permettant à tous nos membres - chercheurs, étudiants et assistants de recherche, mais aussi aux éventuels collaborateurs, de colliger en temps réel et de façon simultanée les données provenant de nos archives. Ainsi, notre base de données nous permet d'accéder à nos données peu importe le lieu géographique où nous nous trouvons.

Une autre opportunité importante qu'offre notre base de données: le partage contrôlé de nos données à des chercheurs nationaux et internationaux qui travaillent sur différentes facettes du suicide. Initialement, notre projet ne devait permettre que d'étudier des thèmes spécifiques à nos intérêts de recherche (suicides migratoires ${ }^{30}$, suicides dans les institutions, etc.), ce qui facilitait l'élaboration de catégories d'analyse. Avec notre intention de rendre nos données accessibles à terme à la commu-

27. Cette période est celle pendant laquelle les dossiers sont accessibles au public. Avec la loi de 1983 (qui entre en vigueur en 1986), le mandat du coroner change et les données ne sont plus publiques. L'enquête du coroner l'est toujours, mais elle se retrouve à l'intérieur d'une chemise qui contient également le rapport de police, rapport qui est confidentiel.

28. À cet égard, il importe de souligner que pour retrouver ces milliers de dossiers de suicide, nous devons d'abord examiner la totalité des fonds d'archives susmentionnés, lesquels contiennent l'ensemble des enquêtes du coroner portant sur des décès variés, décès qualifiés de violents, naturels ou accidentels. Les suicides entrent dans la catégorie «décès violents» selon la lecture faite par le Bureau du coroner.

29. Ce sont les types de documents les plus souvent présents au dossier mais ce n'est pas systématique.

30. Par suicides migratoires nous entendons tous ces cas de suicide où les personnes ont mis fin à leurs jours loin de chez elles, certaines ayant même parcouru des milliers de kilomètres pour ce faire. 
nauté scientifique, nous avons dû réfléchir aux informations pertinentes à colliger à la suite de la numérisation des dossiers. Or, la richesse de notre corpus rend exponentiel le nombre d'éléments codifiables selon les intérêts de recherche de tout un chacun. Nous avons donc décidé de numériser l'ensemble des documents contenus dans chacune des enquêtes du coroner répertoriées. Ainsi, pour chaque enquête du coroner, il sera possible aux chercheurs d'accéder directement à l'ensemble des documents originaux du dossier. Voici un exemple de fiche à laquelle l'enquête numérisée est attachée sous un format pdf.

\begin{tabular}{|c|c|c|c|c|c|c|}
\hline \multicolumn{7}{|l|}{ Informations sur le dossier } \\
\hline \multicolumn{3}{|l|}{ Champs } & \multicolumn{4}{|l|}{ Valeurs } \\
\hline \multicolumn{3}{|l|}{ Localisation } & \multicolumn{4}{|l|}{$\triangle$ BanQ Vieux-Montréal } \\
\hline \multicolumn{3}{|l|}{ Date du dossier } & \multicolumn{4}{|l|}{30 aôt 1904} \\
\hline \multicolumn{2}{|l|}{ Numéro du dossier } & ? & \multicolumn{4}{|l|}{875} \\
\hline \multicolumn{2}{|l|}{ Contenant } & $?$ & \multicolumn{4}{|c|}{12} \\
\hline \multicolumn{2}{|l|}{ ID de la region } & $?$ & \multicolumn{4}{|c|}{6} \\
\hline \multicolumn{2}{|l|}{ Nom de la région } & $?$ & \multicolumn{4}{|c|}{ Montréal- Montérégie-Laval-Lanaudière-Laurentides } \\
\hline \multicolumn{2}{|l|}{ Tribunal } & $?$ & \multicolumn{4}{|l|}{ Cour des sessions de la paix } \\
\hline \multicolumn{2}{|l|}{ ville (tribunal) } & ? & \multicolumn{4}{|l|}{ Montréal } \\
\hline \multicolumn{2}{|l|}{ fonds } & $?$ & \multicolumn{4}{|l|}{$\mathrm{TL32}, \mathrm{S26,551}$} \\
\hline \multicolumn{2}{|l|}{ Année(s) du fonds } & $?$ & \multicolumn{4}{|l|}{ 1768-1908 } \\
\hline \multicolumn{2}{|c|}{ Dossier disponible en ligne (espace serveur) } & $?$ & \multicolumn{3}{|l|}{ BANQ-Montréal-1904-875.pdf } & ZIP I Liste \\
\hline \multicolumn{7}{|c|}{ Informations sur la personne suicidée } \\
\hline \multicolumn{2}{|l|}{ Champs } & & Valeurs & & & \\
\hline Nom & & ? & Lalonde & & & \\
\hline Prénom & & $?$ & Mary & & & \\
\hline Inconnu(e) & & ? & non & & & \\
\hline Sexe mentionnt & & $?$ & femme & & & \\
\hline Etat civil mentionne & & ? & célibataire & & & \\
\hline No civique de la ressidence & & $?$ & 239 & & & \\
\hline type et nom de rue de la ressi & dence & ? & Saint-Urbain & & & \\
\hline Ville (résidence) & & $?$ & Montréal & & & \\
\hline Suicide migratoire possible & & ? & non & & & \\
\hline Profession ou occupation me & ntionnée & $?$ & employée à la maison Seroggie (n) & & & \\
\hline Circonstances du suicide & & & & & & \\
\hline Champs & & & Valeurs & & & \\
\hline Précisions sur le lieu du suici & & ? & Suicidée a I'hôted & & & \\
\hline Cattgorie du moven de suicl & & $?$ & empoisonnement/intoxication & & & \\
\hline Moyen de suicide mentionné & & & Empoisonnement & & & \\
\hline Constat de décès & & & & & & \\
\hline Champs & & & Valeurs & & & \\
\hline Date du décès constaté & & $?$ & $1904 / 08 / 30$ & & & \\
\hline verdicts & & & & Historique & & \\
\hline Champs & Valeurs & & & Actions & Valeurs & \\
\hline $\begin{array}{l}\text { Intitulé de la fiche } \\
\text { (modèle principal) }\end{array}$ & $1904-08-$ & 0.875 & & création & $\begin{array}{l}26 \text { novembre } 2014 \text { 15:08:07 par } \\
\text { lyonnais_a }\end{array}$ & \\
\hline $\begin{array}{l}\text { Nom et prénom du } \\
\text { coroner }\end{array}$ & Ed. Mac Mah & & & modification & $\begin{array}{l}26 \text { novembre } 2014 \text { 15:08:07 par } \\
\text { lyonnais_a }\end{array}$ & \\
\hline $\begin{array}{l}\text { Verdict tiré de la base } \\
\text { de données Filemaker }\end{array}$ & "s'étant emp & sonné & ée avec du Vert de paris" & validation & cette fiche n'est pas validée & \\
\hline
\end{tabular}


L'interface de la base de données se divise en six sections. Tout d'abord, nous avons 1) les informations générales sur le dossier qui permettent de le localiser, c'est-à-dire la date du dossier, son numéro, le fonds d'archive, etc. Sur cette page d'accueil, on retrouve les différents documents du dossier qui ont été numérisés. Ensuite, nous retrouvons 2) les informations disponibles sur la personne suicidée comme la mention du sexe du défunt, son lieu de résidence, son âge, son état civil, son lieu du travail, etc. Des disparités existent dans ces champs de données: parfois l'âge, l'état civil, le lieu de travail ou autres ne sont pas mentionnés dans les documents disponibles au dossier. À partir des années 1970, l'uniformisation des données recueillies par le coroner rend l'entrée systématique des données plus facile ${ }^{31}$. La troisième section porte sur 3) les circonstances entourant le décès, par exemple la date du geste suicidaire, le ou les moyens utilisés, le lieu du suicide, alors que la quatrième se concentre sur 4) les éléments liés au constat du décès, c'est-à-dire la date, le lieu et l'autorité ayant constaté le décès, etc. Les deux dernières sections nous informent sur 5) le verdict de l'enquête et 6) les pièces présentes aux dossiers. Nous avons à cet égard conçu la base de données afin de pouvoir décortiquer l'ensemble des éléments informatifs contenus dans les dossiers, que ce soit les rapports d'autopsie, les rapports de professionnels (policier, gardien de prison, psychiatre, etc.), les différents témoignages ou encore les lettres d'adieu laissées par le défunt. Cette démarche, bien que laborieuse, permettra au final de tenir compte de la pluralité des voix qui se font entendre lorsqu'un décès répertorié comme suicide par les acteurs survient.

L’ensemble de ces données nous permettra d'étudier la répartition des suicides par catégories d'âge, par moyens utilisés, par lieu de décès, par types de "troubles mentaux allégués», etc. De plus, les adresses des suicidés, lorsqu'elles sont mentionnées, ont été entrées dans la base de données de façon à permettre un "géo-référencement» des suicides sur le territoire québécois. Comme nous le disions précédemment, la richesse de ces données ne doit pas nous faire perdre de vue que les informations recueillies sur un temps si long (depuis 1763) obligent à la prudence dans les analyses. Par exemple, une municipalité au tournant du $\mathrm{XX}^{\mathrm{e}}$ siècle est devenue un arrondissement au cours des dernières années. Il en va de même pour l'attribution du qualificatif "vieux» au XIX ${ }^{e}$ siècle qui ne correspond pas à la catégorie du $3^{\mathrm{e}}$ âge dans les années 1980. Le même constat s'applique concernant les appellations pour décrire la présence ou 
non de troubles mentaux chez l'individu: la «mélancolie» d'hier est-elle devenue la "dépression» d'aujourd'hui ${ }^{32}$ ? Bref, les changements dans l'appellation des lieux, de l'âge et des troubles psychiques notamment nous forcent à construire notre base de données de manière intégrative, c'est-à-dire que nous transcrivons les données telles que mentionnées au dossier, tout en créant un espace de regroupement des énoncés que le chercheur pourra interroger et interpréter.

\section{ENJEUX MÉTHODOLOGIQUES ET LIMITES DES DONNÉES RECUEILLIES}

Les milliers de cas repérés dans les enquêtes du coroner à Bibliothèque et Archives nationales du Québec (BAnQ) et numérisés dans notre base de données ne constituent pas la totalité des cas de suicide survenus au Québec depuis 1763. Alors que certains ont pu être "oubliés» (disparition du corps par exemple), d'autres ont pu être étiquetés différemment (décès accidentel, intoxication, etc.). En effet, si certaines variables permettent d'identifier plus facilement un décès par suicide (mort par pendaison ou lorsqu'une lettre d'adieu est retrouvée), d'autres sont sujettes à de possibles interprétations (une chute, une noyade ou une surdose). Il en va de même pour des comportements que nous pourrions définir comme autodestructeurs (consommation intensive de drogues, anorexie qui conduit à la mort, arrêt délibéré de médicamentation, etc.). Autrement dit, un comportement doit-il impérativement avoir un effet létal à court terme pour se voir attribuer l'étiquette de suicide? Mentionnons qu'il est également possible de retrouver des faux positifs dans nos données, soit des décès accidentels ou des homicides étiquetés comme des suicides.

La part d'interprétation dans le verdict transparaît également selon l'histoire de vie de la personne décédée. Ainsi, les individus avec un parcours de vie marqué par de multiples tentatives de suicide, ceux qui souffrent de troubles mentaux (dépression, par exemple) ou encore ceux qui ont des problèmes de dépendance semblent recevoir plus facilement un verdict clairement établi de suicide. Or il serait par exemple tout à fait possible qu'une personne intoxiquée ou dépressive puisse être décédée par accident et non volontairement. Encore une fois, il ne s'agit pas pour nous de nier les corrélations qui existent entre ces différents facteurs et le geste suicidaire. Il s'agit plutôt ici de rappeler que ces décès sont interprétés par des acteurs du système judiciaire et que ceux-ci sont des acteurs

32. Pour plus d'information sur l'histoire des classifications psychiatriques, voir Peter Conrad et Joseph W. Schneider, Deviance and Medicalization: from Badness to Sickness (Philadelphie, Temple University Press, 1991); Isabelle Perreault, Psychiatrie et ordre social, Thèse de doctorat (histoire), Université d'Ottawa, 2009. 
OUE LES RECMERCHES FAITES ETABLISENT OUE LA MORT EST DUEA
THAT THE INVESTIGATIONS CARAIEO OUT HAVE ESTABLISHED THAT

Violente Sulcide probable

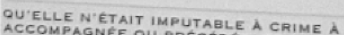

ACCOMPAGNEE OU PRECEDEE, ET OUE A OUI QUE CE SOIT, NI A LA NEGLIOENCE OE PEF

DEATH WAS NOT THE RESULT OU IL N'Y AVAIT PAS LIEU OE LENER UNIGENCE DE PERSONNE: QUUAUCUN CRIME NE L'AVAIT ACCOMPANIED OR PRECEOED OK CATMINAL ACTION OR NEGLLEENT CONDUCTON

Source: BAnQ,Archives du coroner, dossier n²965, 1969.

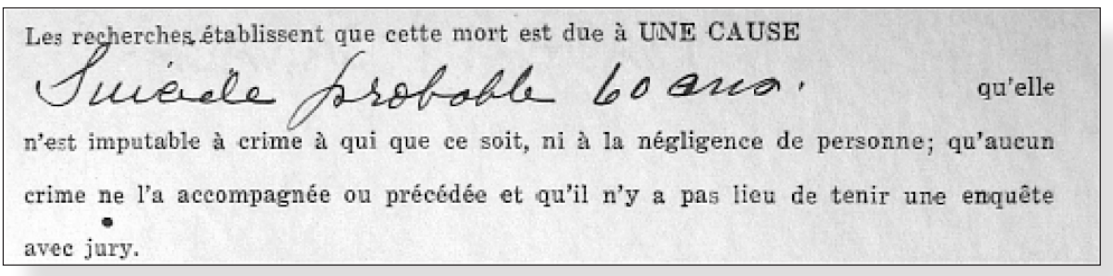

Source: BAnQ,Archives du coroner, dossier n 1559, 1933.

sociaux et moraux avant tout. À ce chapitre, notons que certains verdicts du coroner signalent la part d'incertitude qui persiste à l'égard d'un décès déclaré «suicide probable» ou encore «mort douteuse »"

Une autre limite de notre base de données est liée au caractère tabou du suicide. Les conséquences pour la famille de voir un de ses proches reconnu "coupable» de s'être suicidé (homicidé soi-même jusqu'au XIX ${ }^{e}$ siècle) étaient souvent graves. Les familles des suicidés risquaient de subir des conséquences financières et sociales importantes et le chercheur ne doit donc pas exclure la possibilité que des cas de "suicides » aient été présentés différemment aux autorités afin d'éviter l'opprobre de la collectivité (et les conséquences économiques qui pouvaient en découler). Par exemple, en 1940, la revue Assurances souligne que le suicide ne peut être reconnu par l'assurance puisqu'il vient "fausser les règles du jeu », c'est-à-dire les calculs actuariels liés au risque de décès accidentel ou naturel ${ }^{34}$. Que ce soit la personne elle-même qui souhaite éviter la disgrâce sur sa famille, ou la famille qui explique la mort comme un triste accident, il est fort possible que des morts volontaires se soient retrouvées étiquetées comme des décès accidentels $^{35}$. Certains coroners pourront même hésiter à rendre un verdict de

33. Pour ces cas «limites», une base parallèle à la base de données sera mise sur pied. Photos : BAnQMontréal, Fonds TP12, S2, SS26, 1933, dossier n 1559 et BAnQ-Montréal, Fonds TP12, S2, SS26, 1969, dossier n ${ }^{\circ} 2965$.

34. Revue Assurances, Montréal, 2 (juillet 1940): 61-65.

35. En ne laissant pas de lettre d'adieu et en masquant son geste, par exemple. Une phase de dépouillement préliminaire des archives du coroner à Rimouski (région de Kamouraska, Rivière Ouelle, Rivière du Loup et Cabano dans la $2^{\mathrm{e}}$ moitié du XIX siècle) nous invite à penser plus en avant cette hypothèse. En effet, il y aurait une sous-représentation statistique des décès par suicide au XIX ${ }^{e}$ siècle pour ces 


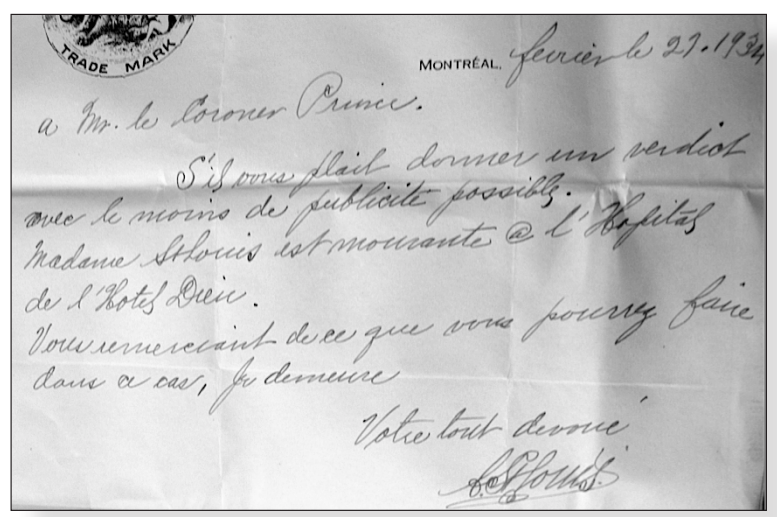

Source: BAnQ, Archives du coroner, dossier n 369, 1934.

suicide dans certaines circonstances afin d'éviter des conséquences désagréables/ déshonorantes, notamment quand la personne suicidée demande explicitement un verdict avec le moins de publicité possible ${ }^{36}$.

En somme, il faut retenir des limites méthodologiques inhérentes à notre recherche que les cas répertoriés sont d'abord considérés comme suicide par le coroner ou les jurés pour ensuite devenir des suicides lors du dépouillement de ces enquêtes par notre équipe. En d'autres mots, il faut dans un premier temps que les experts appelés à enquêter sur un décès in situ le déclarent officiellement comme étant dû à un acte qui réfère explicitement au fait de s'enlever la vie et dans un deuxième temps que les membres de notre équipe, lors du dépouillement des plumitifs et des archives, identifient eux aussi les dossiers de cette catégorie large de décès par suicide. Il faut se rappeler ici qu'il y a eu diverses manières de nommer le fait de se donner la mort au cours des derniers 250 ans. Dans un registre épistémologique différent du nôtre, notre imposante base de données (pour rappel, plus ou moins 20000 dossiers de suicide) permettra également aux chercheurs qui le souhaitent de procéder à des études de corrélations sur le temps long entre plusieurs facteurs sociologiques, économiques ou démographiques.

régions, car la cause du décès n’est pas «clairement» établie par le jury. Contrairement aux enquêtes de Montréal, par exemple, dans lesquelles on retrouve souvent la mention suicide, dans le Bas du Fleuve on y décrit succinctement la manière dont la personne est décédée. Prenons en exemple deux dossiers: A. S. est décédé le 5 septembre 1878 à Saint-Modeste. Âgé de 19 ans, sa mort a été causée par «la décharge d'un fusil qu'il tenait à la main». À Sainte-Anne, le 12 janvier 1872, P. C. est décédé "suite à un empoisonnement par de l'acide carbolique qu'il avait bu par accident à bord du navire Three Belts en naufrage sur les battures de Sainte-Anne». BAnQ-Rimouski, Fonds TP9, S4, SS26, 1878, dossier no 14 et BAnQRimouski, Fonds TP9, S4, SS26, 1872, dossier no 2.

36. Photo: BAnQ-Montréal, Fonds TP12, S2, SS26, 1934, dossier n 369. 


\section{CONCLUSION}

Le développement d'une telle base de données accessible par Internet, laquelle sera ouverte d'ici quelques années aux chercheurs de diverses disciplines qui en feront la demande, est possible grâce au partenariat que notre équipe de recherche Une sociologie historique du suicide au Québec (SHSQ) de l'Université d'Ottawa a conclu avec le Réseau sur le suicide, les troubles de l'humeur et les troubles associés (RQSHA) ${ }^{37}$ et le Laboratoire d'analyse historique des régulations sociales (LAHRS) de l'UQAM ${ }^{38}$.

Une fois complétée, cette base de données permettra l'analyse de centaines de milliers de témoignages des principaux acteurs appelés à décrire, commenter et expliquer les suicides entre 1763 et 1986, que ce soit les parents, les amis, les officiers de justice ou les médecins qui ont témoigné dans le cadre d'une enquête du coroner. Nous disposerons également, dans une large mesure, des derniers mots des principaux concernés, les suicidés eux-mêmes, par l'entremise des lettres qu'ils ont laissées avant de mourir ${ }^{39}$. La diversité, la qualité et la quantité des données disponibles permettront de faire des études sociologiques liant ce comportement individuel au contexte social ambiant, mais aussi des analyses préconisant une approche où le suicide est étudié à partir de milliers de cas singuliers ${ }^{40}$.

Au cours des prochaines années, certaines pistes de recherche seront davantage explorées. À l'aide des centaines de milliers de témoignages recueillis par le coroner, nous étudierons tout d'abord les transformations dans les mentalités et attitudes des Québécois qui ont présidé à la mutation de la réaction sociale à l'égard du comportement suicidaire, comportement qui est apparu selon les contextes et les époques comme crime, blasphème, folie, problème de société ou libre choix. Nous examinerons ainsi les éléments du contexte social ambiant qui ont pu avoir une influence sur la modification constatée des attitudes et des comporte-

37. Un grand merci à Alain Lesage, Gustavo Turecki, Monique Séguin et Luc Desrochers de nous avoir ainsi approchés et offert leur support financier et organisationnel.

38. Tout cela n'aurait pu voir le jour sans deux des trois cofondateurs du Centre d'histoire des régulations sociales (CHRS) de l'UQAM, feu Jean-Marie Fecteau et André Cellard. Fecteau nous a offert l'expertise technique et technologique de son équipe lors de la mise en place de notre recherche en 2008 et André Cellard a été à l'origine du présent programme de recherche sur le suicide. Merci également à Martin Petitclerc, actuel directeur, pour avoir poursuivi cette collaboration.

39. Nous avons constaté que plusieurs lettres ne se retrouvent pas dans les dossiers où il est fait mention de leur présence. Par exemple, plusieurs lettres des années 1970 et 1980, étudiées par Éric Volant, Adieu la vie..., op. cit., sont introuvables.

40. Telles que celles mises en œuvre par Émile Durkheim (1897), Bayet (1922) ou Halbwachs (1930) qui ont fait du suicide un objet d'étude sociologique. Voir aussi Jean Baechler, Les suicides (Paris, CalmannLevy, 1975) ou Jack D. Douglas, The Social Meaning of Suicide..., op. cit. 
ments à l'égard des gens qui s'enlevaient volontairement la vie. Nous souhaitons également étudier les causes présumées du suicide selon l'entourage et les officiers de justice. Sont-elles identiques aux raisons exprimées par les suicidés dans leur lettre d'adieu? Nous analyserons à cet égard les types d'arguments (théologique, médical, social, etc.) utilisés par les différents acteurs concernés par le geste suicidaire pour l'expliquer, que ce soit les autorités, l'entourage du suicidé ou le suicidé lui-même.

D’ailleurs, une attention particulière sera donnée aux lettres des suicidés. D'une part, nous souhaitons mettre en lumière les différents motifs évoqués par les suicidés pour mettre fin à leurs jours (détresse psychologique, maladie, problème financier etc.), tout comme les diverses intentions qui traversent leurs mots d'adieu (vengeance, pardon, explication, etc. $)^{41}$. D'autre part, nous nous intéressons également à la mise en scène de soi lors des derniers moments de vie. À l'aide des mots contenus dans les lettres, des photos prises par le coroner ou la police ou encore de la description du corps sur le lieu du suicide, nous étudions les diverses significations symboliques du passage à l'acte comme exercice de performativité.

Par ailleurs, nous avons déjà analysé et poursuivons notre analyse sur les cas de suicide qui se sont produits dans les institutions d'enfermement, que ce soit les prisons, les pénitenciers ou encore les asiles psychiatriques. Par exemple, nous souhaitons comprendre comment les acteurs qui travaillent au sein de ces institutions perçoivent le geste suicidaire, quels rôles le coroner et les témoins leur attribuent-ils dans le passage à l'acte, etc.

Nous travaillons également sur le thème des suicides migratoires évoqués précédemment. L’ampleur de notre corpus empirique nous a en effet permis de déceler ces suicides qui sont commis loin du lieu de résidence des suicidés; par exemple, ces suicides d'immigrants fraîchement arrivés à Montréal qui laissent supposer que le caractère tabou du geste suicidaire pouvait inciter certaines personnes à mettre fin à leurs jours loin de chez eux afin d'éviter l'opprobre ou le déshonneur à leur famille.

Enfin, cette base de données permettra aux chercheurs de diverses disciplines de jeter des lumières nouvelles, même discrètes sur les raisons sociales et individuelles qui ont conduit à une augmentation de la prévalence des suicides dans la société québécoise ainsi qu’à mieux saisir la transformation du profil démographique de ceux qui les commettent.

41. Un article portant sur des lettres de suicide paraitra en 2016: Isabelle Perreault, Patrice Corriveau et Jean-François Cauchie, "While of Unsound Mind? Narratives of Responsibility in Suicide Notes in the $20^{\text {th }}$ Century", Histoire sociale/Social History. 